\title{
The Simplex algorithm for the rapid identification of operating conditions during early bioprocess development: case studies in FAb' precipitation and multimodal chromatography
}

\begin{tabular}{|r|l|}
\hline Journal: & Biotechnology and Bioengineering \\
\hline Wanuscript ID: & Draft \\
\hline Datey - Manuscript type: & Article \\
\hline Author: & n/a \\
\hline Complete List of Authors: & $\begin{array}{l}\text { Chhatre, Sunil; University College London, The Advanced Centre for } \\
\text { Biochemical Engineering } \\
\text { Konstantinidis, Spyridon; University College London, The Advanced } \\
\text { Centre for Biochemical Engineering } \\
\text { Ji, Yu; University College London, The Advanced Centre for } \\
\text { Biochemical Engineering } \\
\text { Edwards-Parton, Simon; University College London, The Advanced } \\
\text { Centre for Biochemical Engineering } \\
\text { Zhou, Yuhong; University College London, The Advanced Centre for } \\
\text { Biochemical Engineering University College London, The Advanced } \\
\text { Titchener-Hooker, Nigel; Univer } \\
\text { Centre for Biochemical Engineering }\end{array}$ \\
\hline Key Words: & $\begin{array}{l}\text { Design of Experiments (DoE), FAb' precipitation, Factorial Design, } \\
\text { Polyclonal antibody, Multimodal chromatography, Simplex algorithm }\end{array}$ \\
\hline
\end{tabular}

\section{SCHOLARONE" Manuscripts}




\section{The Simplex algorithm for the rapid identification of operating conditions during early bioprocess development: case studies in FAb' precipitation and multimodal chromatography}

Sunil Chhatre, Spyridon Konstantinidis, Yu Ji, Simon Edwards-Parton, Yuhong Zhou and Nigel J. Titchener-Hooker*

The Advanced Centre for Biochemical Engineering, Department of Biochemical Engineering, University College London, Torrington Place, London, United Kingdom.

WC1E 7JE; (* telephone: +44 207679 3796; facsimile: +44 207916 3943; e mail: nigelth@ucl.ac.uk)

Running title: Simplex algorithm for bioprocess development 
Keywords: Bioprocess development, Design of Experiments (DoE), FAb' precipitation,

Factorial Design, Polyclonal antibody, Multimodal chromatography, Simplex algorithm

\begin{abstract}
This paper describes a data-driven algorithm as a rapid alternative to conventional Design of Experiments (DoE) approaches for identifying feasible operating conditions during early bioprocess development. In general, DoE methods involve fitting regression models to experimental data, but if model fitness is inadequate then further experimentation is required to gain more confidence in the location of an optimum. This can be undesirable during very early process development when feedstock is in limited supply and especially if a significant percentage of the tested conditions are ultimately found to be sub-optimal. An alternative approach involves focusing solely upon the feasible regions by using the knowledge gained from each condition to direct the choice of subsequent test locations that lead towards an optimum. To illustrate the principle, this paper describes the application of the Simplex algorithm which uses accumulated knowledge from previous test points to direct the choice of successive conditions towards better regions. The method is illustrated by two case studies; a two variable precipitation example investigating how salt concentration and $\mathrm{pH}$ affect $\mathrm{FAb}$ ' recovery from $E$. coli homogenate and a three-variable chromatography example identifying the optimal $\mathrm{pH}$ and concentrations of two salts in an elution buffer used to recover ovine antibody bound to a multimodal cation exchange matrix. Two-level and face centred central composite regression models were constructed for each study and statistical analysis showed that they provided a poor fit to the data, necessitating additional experimentation to confirm the robust regions of the search space. By comparison, the Simplex algorithm identified a good operating point using $50 \%$ and $70 \%$ fewer conditions for the precipitation and chromatography studies respectively. Hence data-driven approaches have significant potential for early process development when material supply is at a premium.
\end{abstract}




\section{INTRODUCTION}

In recent years, high-throughput screening (HTS) technologies have yielded huge increases in productivity for early bioprocess development, allowing manufacturing insight to be gained from microlitre feed volumes (Micheletti and Lye, 2006; Titchener-Hooker et al., 2008) and helping to direct the focus of subsequent development activities. Efficient implementation of HTS requires both suitable DoE approaches to provide appropriate structure to the studies and prior process knowledge to limit design variables to their most relevant ranges (Lundstedt et al., 1998; Olsson et al., 2006; Mandenius and Brundin, 2008; Jiang et al., 2010). A typical DoE approach starts with a screening design such as a two-level factorial with centre points to provide preliminary insight and if curvature is found, the design can be augmented to a response surface method (RSM) to characterise useful regions in more depth. DoE relies upon regression to model the data and statistical analysis to determine the closeness of fit. Where fitness is found to be inadequate, models will provide only a poor approximation of an optimum and additional test conditions are then needed to identify a good operating location. This can be difficult during early development if many conditions need to be tested since only very small feed volumes are available at this stage. Furthermore if many conditions turn out to be sub-optimal then the feed consumed in those experiments will ultimately be wasted, representing a significant loss of a scarce and highly valuable resource. Hence anything which can be done to reduce the total quantity of experimentation in early development is beneficial. One way to achieve this involves maximising the value extracted from every test point by using an experimental design that deliberately searches for favourable regions in a search space over the course of an experimental campaign. One such design is the Simplex algorithm (Nelder and Mead, 1965; DiBella et al., 2008), which is a hill-walking method that uses defined mathematical manipulations to select successive conditions that step away from sub-optimal locations towards superior regions. Hence 
central to our proposition is that although the two-level-response surface route described above offers a high probability of identifying the global robust optimum region, this can potentially require a larger amount of experimentation than the Simplex method, which instead provides a viable local optimum point in return for less laboratory effort. Making this trade-off during early-stage development provides researchers with useful information that can help to target subsequent experimental effort upon the most promising manufacturing strategy. To illustrate the utility of the Simplex method, this paper compares it with a twolevel - RSM approach for two bioprocess case studies, as described below.

\section{METHODOLOGY}

\section{The Simplex algorithm}

Overview

In this paper, the sequential modified Simplex algorithm was used (Dantzig, 1963; Lagarias et al., 1998; Nelder and Mead, 1965). Although the algorithm was developed originally for numerical optimisation of mathematical functions, the technique can also identify good experimental conditions using accumulated data generated at discrete parameter levels. The algorithm is iterative, with the understanding gained after testing each condition enabling selection of subsequent test locations. The mathematical basis of the algorithm is the formation of a simplex within the variable space e.g. a $2 \mathrm{D}$ simplex is a triangle and a $3 \mathrm{D}$ simplex is a pyramid. Each corner of the simplex represents one combination of input variable values and an objective function is used to quantify performance at each corner in terms of a desired outcome (e.g. to maximise yield). The algorithm uses values from previous experimental steps to construct simplices that move away progressively from the worst points towards better conditions with superior objective function values. Evolution in 


\section{Implementation of the method}

For a search space with $n$ variables, the initial simplex contains $n+1$ corner points and rules governing how this shape is manipulated at each iteration (Walters et al., 1999) are shown graphically in two dimensions in Figure 1. In silico manipulations take the form of reflection, expansion, contraction and shrinkage and the exact distance by which points move is dictated by numerical coefficients (Lagarias et al., 1998). Although these rules are adequate for optimising mathematical functions, additional measures are required to handle experimental data. For example, limits exist on the smallest size of incremental steps for experimental variables due to inherent equipment imprecision. Thus if the algorithm was to suggest two successive $\mathrm{pH}$ values of 7.00 and 7.02, the results could be unreliable given a typical $\mathrm{pH}$ probe error of \pm 0.10 and hence any actions taken by the algorithm could be erroneous. A minimum step size is needed in silico to prevent generation of impractical test points. Hence the Simplex method was implemented in MATLAB version 7 (The Math Works Incorporated, Massachusetts, U.S.A.) accounting for practical limitations such as the $\pm 0.10 \mathrm{pH}$ tolerance by rounding to the nearest practical value e.g. if the method suggested $\mathrm{pH} 7.02$, this was adjusted to 7.00. To minimise the experimental burden in the case studies described below, one initial simplex was set-up in the middle of the design space and the algorithm was terminated after a total of ten iterations at unique (i.e. hitherto untested) points, which defined the practical time limit set for experimental studies. Although starting with multiple simplex searches improves the chances of identifying an optimum, this increases the experimental burden and may place greater demands on feedstock supply than can be satisfied during early development. 


\section{Case studies}

To illustrate the advantages of the Simplex method, the following sections describe two high throughput robotic case studies. The first is a precipitation example, involving a 96-well plate based determination of the impacts made by salt concentration and $\mathrm{pH}$ on the amount of antibody fragment $\left(\mathrm{FAb}^{\prime}\right)$ precipitated, while the second uses a microscale chromatography pipette tip approach to study the elution conditions for recovering polyclonal antibodies bound to a multimodal cation exchange chromatography resin. In each case, regression models for two-level and face-centred central composite designs (CCF) were constructed using Design Expert version 8 (Stat-Ease Incorporated, Minneapolis, Minnesota, U.S.A.). Reagents were purchased from Sigma-Aldrich (Poole, Dorset, UK).

\section{FAb' precipitation case study}

Feed material preparation

E. coli strain W3110 with plasmid pTTOD A33 expressing FAb' (Balasundaram et al., 2009; Bowering et al., 2002; Tustian et al., 2007) was cultivated in a 10 L Applikon fermenter (Applikon Biotechnology, Tewkesbury, Gloucestershire, U.K.). Cells were harvested at 60 L/hr using a Carr Powerfuge P6 tubular bowl centrifuge (Pneumatic Scale Corporation, Clearwater, Florida, U.S.A.) at 15,000 rpm, resuspended in $0.1 \mathrm{M}$ sodium phosphate at $\mathrm{pH}$ 7.00 and homogenised for two passes at 500 bar using a Gaulin Micron Lab 40 homogeniser (APV Gaulin GmbH, Lubeck, Germany). The homogenate was then centrifuged using an Eppendorf 5810R centrifuge (Eppendorf UK, Cambridge, UK), after which the FAb'enriched supernatant was used for precipitation studies.

\section{$F A b^{\prime}$ precipitation}

Precipitation studies were undertaken in 96 deep-well conical bottom plates (ABgene, Epsom, U.K.) using a MultiProbe II EX robot (Perkin Elmer, Waltham, Massachusetts, 
U.S.A.) fitted with a four channel pipetting arm. $200 \mu \mathrm{L}$ and $1 \mathrm{~mL}$ disposable conductive BioRobotix tips (VWR International, Lutterworth, Leicester, U.K.) were used for liquid handling. Precipitations were carried out using $\mathrm{pH}$ values between 5.00 and 8.00 in 0.50 unit increments and ammonium sulphate at concentrations between 0 and $3 \mathrm{M}$ in $0.3 \mathrm{M}$ increments with a centre point at $\mathrm{pH} 6.5,1.5 \mathrm{M}$ ammonium sulphate. All values were collected in triplicate. These step sizes were above the smallest practically achievable increments, reducing the impact of error in measurement of performance at neighbouring test conditions. Solutions were buffered to the correct $\mathrm{pH}$ using $0.10 \mathrm{M}$ sodium acetate (pH 5.00 - 6.00) and $0.10 \mathrm{M}$ sodium phosphate ( $\mathrm{pH} 6.50-8.00) .150 \mu \mathrm{L}$ of the buffer was pipetted into the plate, followed by a mixture of $3.85 \mathrm{M}$ ammonium sulphate and water in the appropriate proportions for the target salt concentration. $200 \mu \mathrm{L}$ of FAb'-enriched, clarified supernatant was then added to give a total well volume of $1.8 \mathrm{~mL}$. The plate was sealed and mixed at 450 r.p.m. for two hours using an Eppendorf thermomixer set to $25^{\circ} \mathrm{C}$, before being centrifuged at $4000 \mathrm{rpm}$ for 15 minutes in an Eppendorf 5810R centrifuge.

\section{HPLC analysis}

The clarified supernatant was transferred robotically to an Agilent 96-well sample loading plate (Agilent Technologies U.K. Limited, Cheshire, U.K.) for FAb' HPLC analysis conducted on an Agilent 1200 system by loading $100 \mu \mathrm{L}$ of sample on to a $1 \mathrm{~mL}$ Protein $\mathrm{G}$ HiTrap column (GE Healthcare, Buckinghamshire, U.K.) at $2 \mathrm{~mL} / \mathrm{min}$. $0.02 \mathrm{M}$ sodium phosphate $\mathrm{pH} 7.40$ was used for column equilibration and washing. Elution was achieved by $0.02 \mathrm{M}$ sodium phosphate $\mathrm{pH} 2.50$. The $220 \mathrm{~nm}$ elution peaks were integrated and converted into concentrations by a calibration curve. For the purposes of the Simplex algorithm, the goal was to identify conditions which maximised the amount of FAb' in the precipitated solid phase. Hence HPLC concentrations of FAb' remaining in the liquid phase after precipitation 
were multiplied by -1 such that a large quantity of supernatant FAb' resulted in a low objective function value (reflecting the small amount in the precipitate). Correspondingly, a low supernatant FAb' value after multiplication resulted in a high objective function, indicating a large precipitated FAb' concentration.

\section{Polyclonal antibody chromatography case study}

Robotic tip chromatography operation

Crude hyperimmunised ovine serum (Protherics U.K. Ltd, Blaenwaun, Ffostrasol, Llandysul, Wales, U.K.) was used as the polyclonal feed, with an antibody titre of $36 \mathrm{mg} / \mathrm{mL}$ (Chhatre et al., 2010). The material was stored at $-20{ }^{\circ} \mathrm{C}$ in $1.5 \mathrm{~mL}$ lots and after thawing a tube, it was diluted to $10 \mathrm{mg} / \mathrm{mL}$ of antibody using $0.01 \mathrm{mM}$ sodium acetate, $0.01 \mathrm{mM}$ sodium chloride pH 4.75 (Chhatre et al., 2009) and applied to chromatography pipette tips. These were supplied by PhyNexus (San Jose, California, U.S.A.) and were packed with $20 \mu \mathrm{L}$ of the multimodal cation exchange resin Capto MMC (GE Healthcare, Buckinghamshire, U.K) between two frits at the base of each tip. Chromatographic operation was carried out robotically on a Freedom Evo 150 platform containing an eight channel liquid handling arm, a plate manipulator and an InfiniTe 200 plate reader (Tecan, Reading, U.K.). Disposable tip adaptors on the end of each channel enabled Capto MMC tips to be picked up and a 'low DiTi-eject' option was used to place them back into the tip rack after use. The robot was controlled using EVOware version 2.1 software and all chromatography tip pipetting steps were carried out at $15 \mu \mathrm{L} / \mathrm{s}$ using the buffer sequence in Table I. The robot transferred stock buffers from $100 \mathrm{~mL}$ reservoirs (Tecan, Reading, U.K.) into $2 \mathrm{~mL} 96$ deep-square well plates with conical-bottoms (Fisher Scientific U.K. Limited, Loughborough, Leicestershire, U.K.) at $300 \mu \mathrm{L} / \mathrm{s}$ using $1000 \mu \mathrm{L}$ BioRobotix tips (VWR International, Lutterworth, Leicester, U.K.). The feed material was also held in the plates, but it was transferred there manually since the 
serum had been pre-aliquoted in $1.5 \mathrm{~mL}$ lots, making it impractical to hold such a small volume in a $100 \mathrm{~mL}$ trough and transfer it robotically. The volume of buffer/feed used for all steps was $500 \mu \mathrm{L}$. This protocol was then used to investigate how varying the salt composition and $\mathrm{pH}$ of the elution buffer would affect polyclonal antibody yield, as outlined below.

\section{Elution studies}

Initially, the tip binding capacity at a $10 \mathrm{mg} / \mathrm{mL}$ feed concentration was determined in duplicate by applying the feed for a total of eight loading cycles (Table I) and then quantifying the residual antibody concentration in the load well by Protein $G$ analysis (Chhatre et al., 2010). The resulting binding capacity was $37.5 \mathrm{mg} / \mathrm{mL}$, which was close to the $41 \mathrm{mg} / \mathrm{mL}$ value reported previously for polyclonal ovine serum diluted in the same buffer to $5 \mathrm{mg} / \mathrm{mL}$ (Chhatre et al., 2009). On this basis, the study investigated the impact of three elution variables upon antibody yield - namely ammonium sulphate concentration, ammonium chloride concentration and $\mathrm{pH}$. Yield was calculated as the quantity of antibody recovered in the elution aliquot divided by the amount bound during loading (for a 37.5 $\mathrm{mg} / \mathrm{mL}$ capacity and a $20 \mu \mathrm{L}$ tip, this was $0.75 \mathrm{mg}$ ). For the two salts, concentrations between 0 and $1 \mathrm{M}$ in $0.25 \mathrm{M}$ increments were used, while $\mathrm{pH}$ varied between 5.5 and 8.5 in 0.75 unit increments. $0.5 \mathrm{M}$ stock buffer solutions were used to achieve the required $\mathrm{pH}$ : MES (pH 5.50 and 6.25), $\mathrm{Na}_{3} \mathrm{PO}_{4}(\mathrm{pH} 7.00)$ and Tris- $\mathrm{HCl}(\mathrm{pH} 7.75$ and 8.50). Concentrated stock solutions of ammonium sulphate $(3.8 \mathrm{M})$ and ammonium chloride $(4 \mathrm{M})$ were also prepared and poured into separate $100 \mathrm{~mL}$ troughs and placed on the robotic deck. A spreadsheet was set-up to enable automatic calculation of the volumes of salt solutions and water needed to achieve the desired final concentration. The buffer volume of the desired $\mathrm{pH}$ was constant at just under $200 \mu \mathrm{L}$ and calculations were carried out so as to achieve a final 
elution aliquot volume of $500 \mu \mathrm{L}$. These volumes were imported into EVOware and the robot aspirated those quantities from the appropriate troughs and mixed them together in a 96-well plate. The quantity of antibody present in the elution aliquot was determined by Protein $\mathrm{G}$ analysis (Chhatre et al., 2010) and yields were used as the objective function in Design Expert. The centre point at $\mathrm{pH}$ 7.00, 0.50 M ammonium sulphate, 0.50 M ammonium chloride was replicated four times. All experiments were conducted at room temperature.

\section{RESULTS AND DISCUSSION}

\section{FAb' precipitation case study}

The model ANOVA parameters calculated by Design Expert for the two case studies are shown in Table II and the contour plots generated by regression modelling are in Figure 2. The two-level models provided an initial indication of where operation would be feasible. The most favourable precipitation results were achieved using a low $\mathrm{pH}$ and a high ammonium sulphate concentration, with the feasible region forming a curved region extending out towards the extremes of low salt, low $\mathrm{pH}$ and high salt, high $\mathrm{pH}$. Since the ANOVA showed that curvature was significant, the design was augmented to a CCF response surface. The additional points indicated that in fact, the feasible area was constrained predominantly to the highest salt concentration $(3.0 \mathrm{M})$ and was independent of $\mathrm{pH}$. The CCF ANOVA showed that the lack of fit F-value was significant and owing to this uncertainty, it was necessary to characterise the feasible area identified by the CCF design in more depth. This involved a total of twelve conditions with $\mathrm{pH}$ values of 5, 6, 7 and 8 and ammonium sulphate concentrations of 2.4, 2.7 and 3.0 M (Figure 3). Except at pH 5.0, 2.4 M salt, all outputs were consistent across the range, indicating this to be a robust operating region and resulting in a total experimental requirement of nineteen unique conditions. To 
compare this with the Simplex algorithm, a search was initiated at the centre of the precipitation space, using the average of the triplicate responses at each condition to drive algorithm movements. The step size of the input variables was 0.5 and 0.3 for $\mathrm{pH}$ and ammonium sulphate respectively and ten simplex steps were evaluated in total. The movements made by the algorithm are shown in Figure 4. The Simplex search identified a good area within the $\mathrm{pH} 6.0-7.0,2.4-3.0 \mathrm{M}$ salt range and characterised a number of points within that region during iterations five to ten. Figure 5 plots the response value at every iteration and the shift between the third and fourth bar illustrates that although that Simplex step moved in the wrong direction, this prompted the algorithm to change direction and look elsewhere in the search space. The method was then able to find a good area, with the outputs in iterations five to ten being similar to one another and providing some evidence of robustness. The best value was found at $\mathrm{pH} 6.5,3 \mathrm{M}$ salt, which lies within the robust range identified by the nineteen-point DoE run above. Although the size of the robust region is larger in reality than suggested by Simplex, the smaller area still offers sufficient operational flexibility. This is the trade off between depth of information and experimental burden achieved by the Simplex method which tests approximately half the number of conditions required by the two-level - CCF route (Table III).

\section{Polyclonal antibody chromatography case study}

The two-level model for the chromatography study (Figure 2) suggested that the response was primarily $\mathrm{pH}$-independent and that the best operating area was located at $0 \mathrm{M}$ ammonium sulphate, $1 \mathrm{M}$ ammonium chloride. The negative predicted $\mathrm{R}^{2}$ value indicated, however, that the mean of the data set was more useful for predicting the response than the regression model itself (Table II). The ANOVA also showed the presence of significant curvature, again prompting the use of a CCF design. Visual inspection of the resulting plots indicated 
that the feasible region was more strongly dependent upon $\mathrm{pH}$ than the two-level model suggested and although this variable was still less significant than the two salt concentrations, it was noticeable that the feasible region was far larger in the CCF model at $\mathrm{pH} 8.5$ than was the case in the two-level model. Nevertheless, the lack of fit F-value was again significant and the predicted $\mathrm{R}^{2}$ value of 0.2430 was far below the adjusted $\mathrm{R}^{2}$ of 0.8234 . Hence it was necessary again to increase confidence in the feasible areas in the CCF analysis. The more detailed characterisation was undertaken at $0-0.25 \mathrm{M}$ ammonium sulphate, $0.75-1 \mathrm{M}$ ammonium chloride and since there was some uncertainty about the impact of $\mathrm{pH}$, the effect of this variable between 5.5 and 8.5 in 0.75 unit increments was studied. The results in Figure 6 indicate that a yield of approximately $70 \%$ was achieved across all of these conditions and confirmed the robustness of the output values with respect to $\mathrm{pH}$. The total number of conditions tested by this design route was 33 . When the ten-point simplex was applied instead (with a pH step size of 0.75 and an increment for both salts of $0.25 \mathrm{M}$ ), results showed a general upward trend in yield (Figure 7), with a 75\% yield achieved in the final iteration at $\mathrm{pH}$ 7.75, $0 \mathrm{M}$ ammonium sulphate, $1 \mathrm{M}$ ammonium chloride. This position lies within the robust operating area identified by the DoE above. Nearby points found in previous iterations at the same salt concentrations at $\mathrm{pH} 6.25$ and 7.00 also achieved a similar yield $(\sim 70 \%)$, which again suggested that $\mathrm{pH}$ was relatively unimportant. Hence, although the full DoE route provided more information about robustness, the Simplex delivered a viable operating point in approximately $30 \%$ of the number of experiments (Table III).

\section{Implications of the Simplex method}

As described above for the two case studies, the strength of the Simplex approach lies in early process development when trying to define an operating point quickly in a search space. By reducing experimental burden compared with the other DoE methodologies explored in 


\section{CONCLUSIONS}

Generating process insight during the early stages of development can be helpful in targeting subsequent activities towards the most promising manufacturing options. To achieve this requires one to minimise the amount of experimental material consumed per test condition, since typically little is available during initial studies. Choosing an experimental design such as the Simplex algorithm which selects test conditions that progressively head towards a good operating location can be an efficient way of achieving this goal. This paper compared two different design routes - one based on a conventional factorial design route and the other using the Simplex algorithm - and illustrated their use by application to FAb' precipitation and multimodal polyclonal antibody chromatography case studies. Whereas the conventional DoE identified a robust optimal region in both cases, the Simplex identified a suitable operating point using $50 \%$ and $70 \%$ fewer points for the precipitation and chromatography examples respectively. Hence the Simplex algorithm is best deployed during early development as a way of identifying a suitable operating point to guide subsequent experimentation. 


\section{ACKNOWLEDGEMENT}

The support of the Engineering and Physical Sciences Research Council Innovative Manufacturing Research Centre (IMRC) in Bioprocessing is acknowledged gratefully. The IMRC is part of The Advanced Centre for Biochemical Engineering, Department of Biochemical Engineering, University College London, with collaboration from a range of $\begin{array}{llll}\text { academic } & \text { partners } & \text { and }\end{array}$ (http://www.ucl.ac.uk/biochemeng/industry/imrc).

\section{REFERENCES}

Balasundaram B, Nesbeth D, Ward JM, Keshavarz-Moore E, Bracewell DG. 2009. Step Change in the Efficiency of Centrifugation Through Cell Engineering: Co-Expression of Staphylococcal nuclease to Reduce the Viscosity of the Bioprocess Feedstock, Biotechnol Bioeng 104: 134-142.

Bowering LC, Bracewell DG, Kesharvarz-Moore E, Hoare M, Weir ANC. 2002. Comparison of Techniques for Monitoring Antibody Fragment Production in E. coli Fermentation Cultures, Biotechnol Progr 18: 1431-1438.

Chhatre S, Bracewell DG, Titchener-Hooker NJ. 2009. A microscale approach for predicting the performance of chromatography columns used to recover therapeutic polyclonal antibodies, J Chromatogr. A, 1216: 7806-7815.

Chhatre S, Francis R., Bracewell DG, Titchener-Hooker NJ. 2010. An automated packed Protein $\mathrm{G}$ micro-pipette tip assay for rapid quantification of polyclonal antibodies in ovine serum, Journal of Chromatography B, 878: 3067-3075 
Dantzig G.B. 1963. Linear Programming and Extensions, Princeton University Press, Princeton, NJ.

DiBella E, Meacle F, Alred P, Case Study: Fitting a Difficult Monoclonal Antibody into a Platform Process while Maintaining Timelines, poster in the Recovery of Biological Products XIII, Quebec City, Quebec, Canada 22-27 June 2008

Jiang C, Flansburg L, Ghose S, Jorjorian P, Shukla AA. 2010. Defining Process Design Space for a Hydrophobic Interaction Chromatography (HIC) Purification Step: Application of Quality by Design (QbD) Principles, Biotechnol Bioeng, 107:985-997

Lagarias JC, Reeds JA, Wright MH, Wright PE. 1998. Convergence properties of the NelderMead simplex method in low dimensions, SIAM J Optim 9:112-147

Lundstedt T, Seifert E, Abramo L, Thelin B, Nystrom A, Pettersen J, Bergman R. 1998. Experimental design and optimization, Chemometr Intell Lab 42:3-40.

Mandenius CF, Brundin A. 2008. Bioprocess optimization using design-of-experiments methodology, Biotechnology Progress, 24:1191-1203

Micheletti M, Lye G. 2006. Microscale bioprocess optimisation. Curr Opin Biotechnol 17:611-618.

Nelder JA, Mead R. 1965. A simplex method for function minimization, Computer J 7:308313. 
Olsson IM, Johansson E, Berntsson M, Eriksson L, Gottfries J., Wold S. 2006. Rational DOE protocols for 96-well plates. Chemometr Intell Lab 83:66-74.

Titchener-Hooker NJ, Dunnill P, Hoare M. 2008, Micro biochemical engineering to accelerate the design of industrial-scale downstream processes for biopharmaceutical proteins. Biotechnol Bioeng 100:473-487.

Tustian AD, Salte H, Willoughby NA, Hassan I, Rose MH, Baganz F, Hoare M, TitchenerHooker NJ. 2007. Adapted Ultra Scale-Down Approach for Predicting the Centrifugal Separation Behavior of High Cell Density Cultures, Biotechnol Progr 23:1404-1410.

Walters FH, Morgan SL, Parker LR, Deming SN. 1999. 5: Comments on Fixed-Size and Variable-Size Simplexes, In: Sequential Simplex Optimisation: a technique for improving quality and productivity in research, development, and manufacturing. Florida: CRC Press (licensed electronic reprint by MultiSimplex AB), 128-130. 


\section{Table and figure legends:}

Table I: Sequence of buffer steps used for the polyclonal antibody tip chromatography study

Table II: ANOVA parameters calculated by Design Expert; no data transformations were employed for modelling. Statistically significant curvature and lack of fit were found in both studies.

Table III: Summary of the number of unique conditions required in each experimental design. Values given for the CCF and detailed characterisation represent the number of new conditions that had not been tested in previous designs.

Figure 1: Logical rules used to step the simplex through a 2D design space (Nelder and Mead, 1965; Lagarias et al., 1998; Walters et al., 1999). The rules can be extended for cases with more than two variables. The '> 'symbol denotes 'superior to.'

Figure 2: Contour maps generated by the regression models for the precipitation and chromatography data. The precipitation responses indicate the supernatant FAb' concentration; chromatography responses indicate antibody elution yield

Figure 3: Detailed characterisation results of the feasible area in the precipitation study. The bars show the average values of triplicate responses measured at each condition. For ease of comparison, all bars have been normalised to the smallest response in the original data set (at pH 7, $0.3 \mathrm{M}$ salt).

Figure 4: Simplex results from the precipitation study (the average of the triplicate responses at each condition was used to drive the search). One initial simplex was established in the centre of the search space (indicated by hollow circles at pH 6.5, 1.8 M; pH 6.0, 1.2 M; pH 7.0, 1.2 M) and seven further iterations were conducted to give the ten locations specified in the diagram.

Figure 5: Response value at every iteration during the Simplex run for the precipitation study. For ease of comparison, all bars have been normalised to the smallest response in the original data set (at pH 7, 0.3 $M$ salt). The first three bars indicate the outputs at the corners of the initial simplex in order of increasing normalised supernatant FAb' concentration.

Figure 6: Detailed characterisation of the feasible area in the chromatography study.

Figure 7: Response value at each iteration during the Simplex run for the chromatography study. The locations of the initial simplex corner points (the outputs plotted in the first four bars) were chosen randomly around the centre of the search space at the following conditions $\left(\mathrm{pH},\left(\mathrm{NH}_{4}\right)_{2} \mathrm{SO}_{4}, \mathrm{and}_{\mathrm{NH}} \mathrm{Cl}\right.$ respectively): (1) 7.00, 0.75 M, 0.25 M; (2) 7.75, 0.75 M, 0.75 M; (3) 6.25, 0.50 M, 0.25 M; (4) 7.00, 0.25 M, $0.50 \mathrm{M}$ 


\begin{tabular}{|c|c|c|c|}
\hline Step & Buffer/solution & No. of cycles & Details \\
\hline Wash & Water & 3 & To remove storage buffer (20\% ethanol) \\
\hline Equilibrate & $0.01 \mathrm{M} \mathrm{NaAc}, 0.01 \mathrm{M} \mathrm{NaCl} \mathrm{pH} 4.75$ & 3 & Buffer chosen previously (Chhatre et al., 2009) \\
\hline Load & $\begin{array}{l}36 \mathrm{mg} / \mathrm{mL} \text { serum diluted in equilibration } \\
\text { buffer to a } 10 \mathrm{mg} / \mathrm{mL} \text { antibody concentration }\end{array}$ & 8 & $\begin{array}{c}\text { Eight steps were chosen since prior in-house } \\
\text { experience with these tips and feed indicated this } \\
\text { was adequate to achieve equilibrium }\end{array}$ \\
\hline Elute & $\begin{array}{l}\text { Ammonium sulphate, ammonium chloride, } \\
\text { water and buffer of appropriate } \mathrm{pH} \text { (see text) }\end{array}$ & 1 & $\begin{array}{l}\text { Only one cycle was used to accelerate } \\
\text { experimentation and analysis }\end{array}$ \\
\hline Wash & Water & 1 & To remove elution buffer \\
\hline Regenerate & $1 \mathrm{M} \mathrm{NaOH}$ & 5 & To strip the resin clean \\
\hline Wash & Water & 3 & To remove $1 \mathrm{M} \mathrm{NaOH}$ \\
\hline Wash & $20 \%$ ethanol & 3 & $\begin{array}{l}\text { To provide a final cleaning step and to prepare tips } \\
\text { for storage in } 20 \% \text { ethanol }\end{array}$ \\
\hline
\end{tabular}

Table I: Sequence of buffer steps used for the polyclonal antibody tip chromatography study 
1

2

3

4

5

6

7

8

9

\begin{tabular}{|c|c|c|c|c|}
\hline & \multicolumn{2}{|c|}{ Precipitation study } & \multicolumn{2}{|c|}{ Chromatography study } \\
\hline & Two-level & $C C F$ & Two-level & $C C F$ \\
\hline Output metric & \multicolumn{2}{|c|}{ Supernatant FAb' concentration } & \multicolumn{2}{|c|}{ Antibody elution yield } \\
\hline Model type & Two-factor interaction & Quadratic & Three-factor interaction & Quadratic \\
\hline$R^{2}$ & 0.7183 & 0.9526 & 0.9021 & 0.9169 \\
\hline Adjusted $R^{2}$ & 0.6415 & 0.9414 & 0.7309 & 0.8234 \\
\hline Predicted $R^{2}$ & 0.6195 & 0.9232 & -16.5612 & 0.2430 \\
\hline Model F-value & 9.35 & 84.48 & 5.27 & 9.80 \\
\hline Model $P>F$ & 0.0023 & $<0.0001$ & 0.0638 & 0.0019 \\
\hline$\% C V$ & 68.25 & 21.80 & 25.00 & 17.45 \\
\hline Lack of fit F-value & 803.36 & 74.64 & 40.10 & 9.08 \\
\hline Lack of fit $P>F$ & $<0.0001$ & $<0.0001$ & 0.0080 & 0.0495 \\
\hline Most significant terms & $\begin{array}{ll}- & \mathrm{A}-\mathrm{pH} \\
- & \mathrm{B}-\left[\mathrm{NH}_{4} \mathrm{SO}_{4}\right] \\
- & \mathrm{AB}\end{array}$ & $\begin{array}{ll}- & \mathrm{A}-\mathrm{pH} \\
- & \mathrm{B}-\left[\mathrm{NH}_{4} \mathrm{SO}_{4}\right] \\
- & \mathrm{AB} \\
- & \mathrm{A}^{2} \\
- & \mathrm{B}^{2}\end{array}$ & $\begin{array}{ll}- & \mathrm{B}-\left[\left(\mathrm{NH}_{4}\right)_{2} \mathrm{SO}_{4}\right] \\
- & \mathrm{C}-\left[\mathrm{NH}_{4} \mathrm{Cl}\right] \\
- & \mathrm{BC}\end{array}$ & $\begin{array}{ll}- & \mathrm{B}-\left[\left(\mathrm{NH}_{4}\right)_{2} \mathrm{SO}_{4}\right] \\
- & \mathrm{C}-\left[\mathrm{NH}_{4} \mathrm{Cl}\right] \\
- & \mathrm{BC}\end{array}$ \\
\hline
\end{tabular}

Table II: ANOVA parameters calculated by Design Expert; no data transformations were employed for modelling. Statistically significant curvature and lack of fit were found in both studies. 


\begin{tabular}{|l|c|c|}
\hline Design type & Precipitation & Chromatography \\
\hline Two-level & 5 & 9 \\
\hline CCF & 4 & 6 \\
\hline Detailed characterisation & 10 & 18 \\
\hline Total: & $\mathbf{1 9}$ & $\mathbf{3 3}$ \\
\hline Simplex & $\mathbf{1 0}$ & $\mathbf{1 0}$ \\
\hline
\end{tabular}

Table III: Summary of the number of unique conditions required in each experimental design. Values given for the CCF and detailed characterisation represent the number of new conditions that had not been tested in previous designs. 
Figure 1: Logical rules used to step the simplex through a 2D design space (Nelder and Mead, 1965; Lagarias et al., 1998; Walters et al., 1999). The rules can be extended for cases with more than two variables. The ' $>$ ' symbol denotes 'superior to.' $1333 \times 1265 \mathrm{~mm}(96 \times 96 \mathrm{DPI})$ 

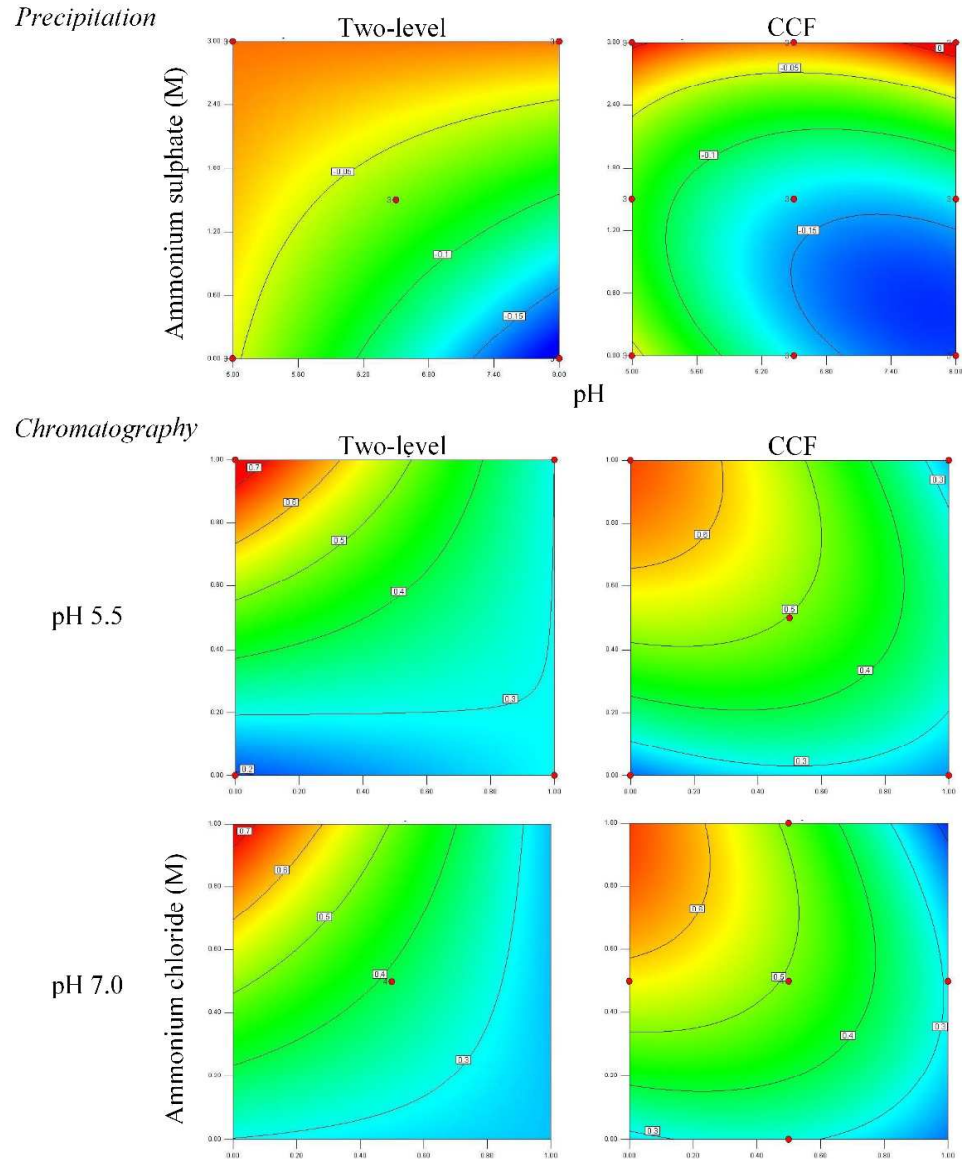

pH 8.5
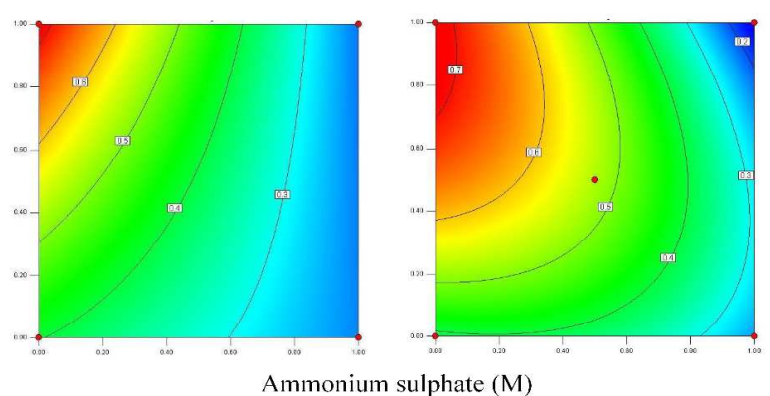

Figure 2: Contour maps generated by the regression models for the precipitation and chromatography data. The precipitation responses indicate the supernatant FAb' concentration; chromatography responses indicate antibody elution yield $910 \times 1492 \mathrm{~mm}(96 \times 96 \mathrm{DPI})$ 


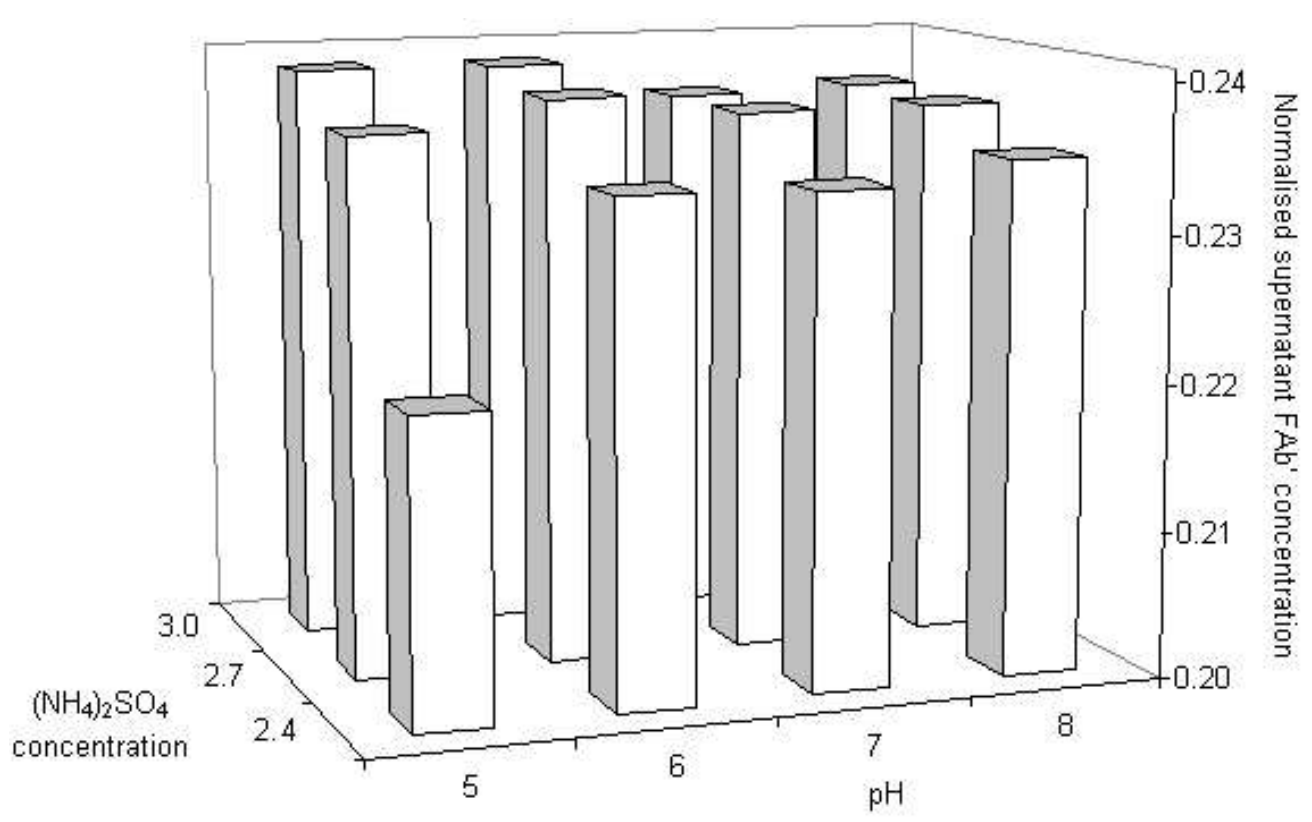

Figure 3: Detailed characterisation results of the feasible area in the precipitation study. The bars show the average values of triplicate responses measured at each condition. For ease of comparison, all bars have been normalised to the smallest response in the original data set (at pH $7,0.3 \mathrm{M}$ salt)

$151 \times 93 \mathrm{~mm}(96 \times 96 \mathrm{DPI})$ 
Figure 4: Simplex results from the precipitation study (the average of the triplicate responses at each condition was used to drive the search). One initial simplex was established in the centre of the search space (indicated by hollow circles at pH 6.5, 1.8 M; pH 6.0,1.2 M; pH 7.0, 1.2 M) and seven further iterations were conducted to give the ten locations specified in the diagram $156 \times 103 \mathrm{~mm}(96 \times 96 \mathrm{DPI})$ 
1

2

3

4

5

6

7

8

9

10

11

12

13

14

15

16

17

18

19

20

21

22

23

24

25

26

27

28

29

30

31

32

33

34

35

36

37

38

39

40

41

42

43

44

45

46

47

48

49

50

51

52

53

54

55

56

57

58

59

60

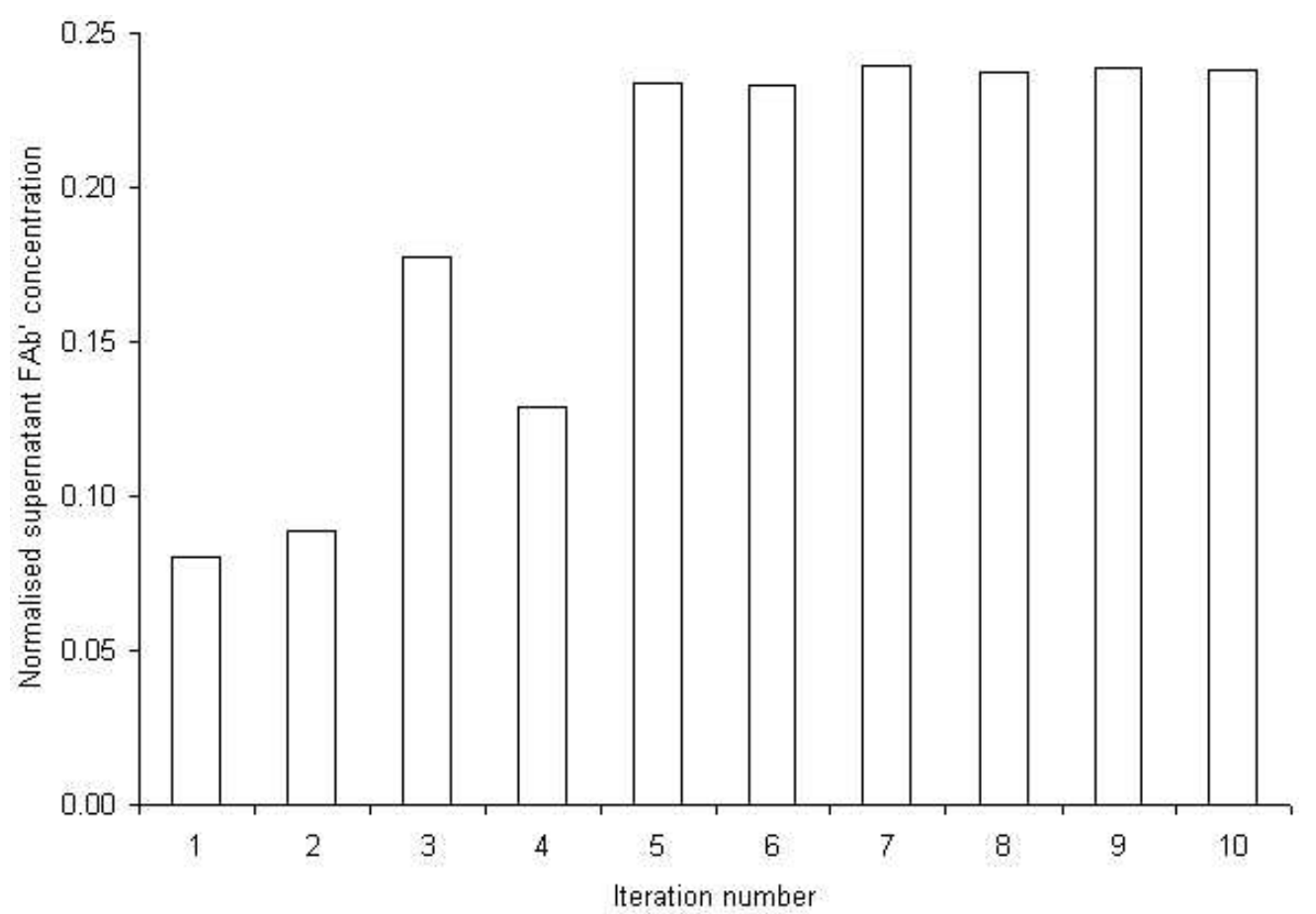

Figure 5: Response value at every iteration during the Simplex run for the precipitation study. For ease of comparison, all bars have been normalised to the smallest response in the original data set (at $\mathrm{pH} 7,0.3 \mathrm{M}$ salt). The first three bars indicate the outputs at the corners of the initial simplex in order of increasing normalised supernatant FAb' concentration $156 \times 109 \mathrm{~mm}(96 \times 96 \mathrm{DPI})$ 
Figure 6: Detailed characterisation of the feasible area in the chromatography study $104 \times 330 \mathrm{~mm}$ (96 x 96 DPI) 
1

2

3

4

5

6

7

8

9

10

11

12

13

14

15

16

17

18

19

20

21

22

23

24

25

26

27

28

29

30

31

32

33

34

35

36

37

38

39

40

41

42

43

44

45

46

47

48

49

50

51

52

53

54

55

56

57

58

59

60

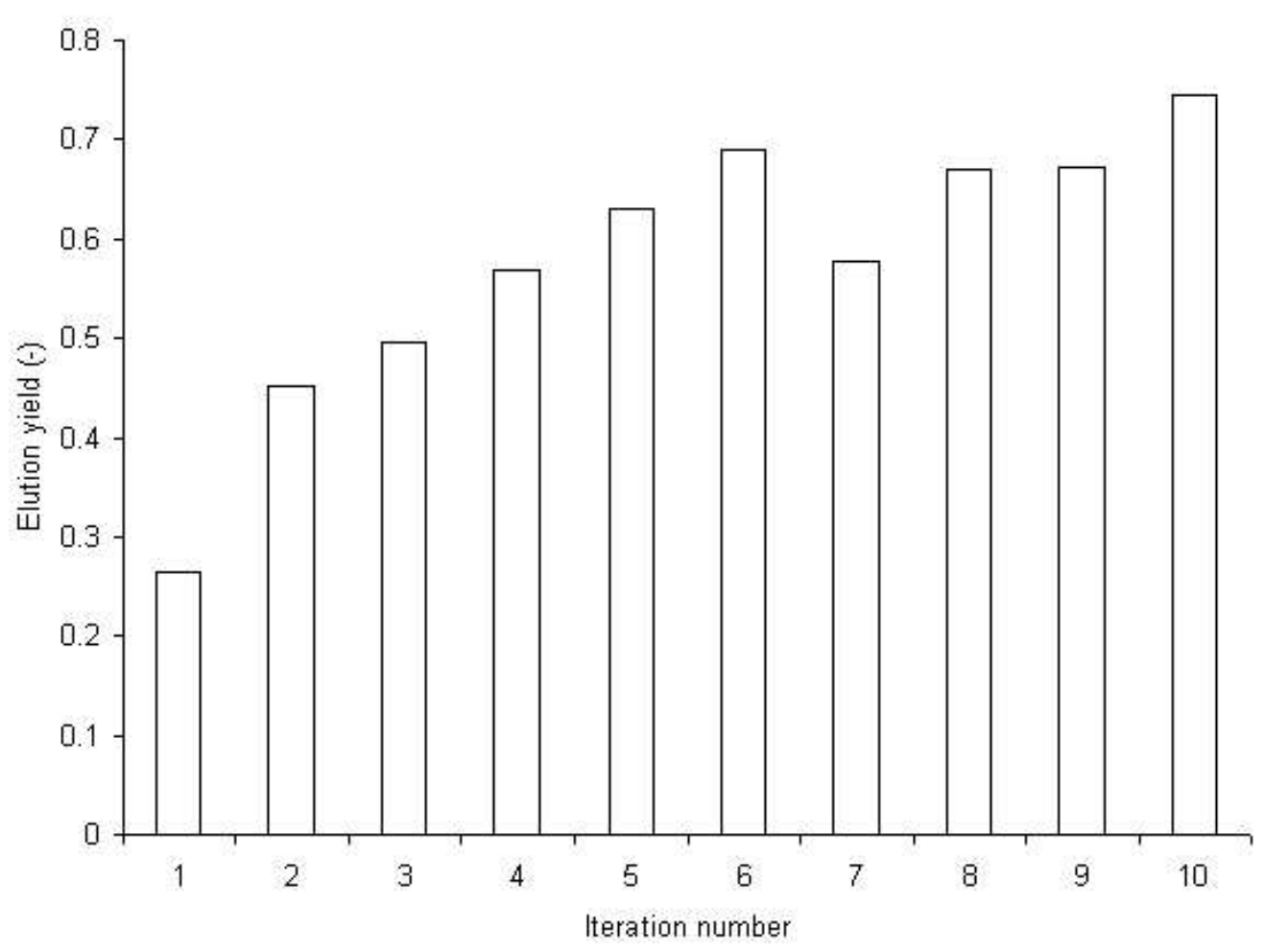

Figure 7: Response value at each iteration during the Simplex run for the chromatography study. The locations of the initial simplex corner points (the outputs plotted in the first four bars) were chosen randomly around the centre of the search space at the following conditions $\left(\mathrm{pH}_{\mathrm{t}}\right.$ (NH4)2SO4, and $\mathrm{NH} 4 \mathrm{Cl}$ respectively): (1) 7.00, $0.75 \mathrm{M}, 0.25 \mathrm{M}$; (2) 7.75, $0.75 \mathrm{M}, 0.75 \mathrm{M}$; (3) 6.25, $0.50 \mathrm{M}, 0.25 \mathrm{M} ;(4) 7.00,0.25 \mathrm{M}, 0.50 \mathrm{M}$ $151 \times 112 \mathrm{~mm}(96 \times 96 \mathrm{DPI})$ 